\title{
CHARACTERISATION OF THE TRIUMF NEUTRON FACILITY USING A MONTE CARLO SIMULATION CODE
}

\author{
Monk S.D. , Abram T., Joyce M.J. \\ Department of Engineering, Lancaster University, Lancaster, United Kingdom LA1 4YR \\ Received month date year, amended month date year, accepted month date year
}

\begin{abstract}
Here, we describe the characterisation of the high-energy neutron field at TRIUMF (The Tri Universities Meson Facility, Vancouver, British Columbia) with Monte Carlo simulation software. The package used is MCNPX version 2.6.0, with the neutron fluence rate determined at three locations within the TRIUMF neutron facility (TNF), including the exit of the neutron channel where users of the facility can test devices which may be susceptible to the effects of this form of radiation. The facility is often used to roughly emulate the field likely to be encountered at high altitudes due to radiation of galactic origin and thus the simulated information is compared to the energy spectrum calculated to be due to neutron radiation of cosmic origin at typical aircraft altitudes. The calculated values were also compared to neutron flux measurements which were estimated using the activation of various foils by the staff of the facility, showing agreement within an order of magnitude.
\end{abstract}

\section{INTRODUCTION}

Radiation fields around high-energy particle accelerators are often of a complex nature, due to the presence of variety of different radiation types spanning wide ranges in energy ${ }^{(1)}$. Of the various radiation types constituting the field found in such an environment, neutrons can present the greatest contribution to dose equivalent ${ }^{(2)}$. TRIUMF (the TRI Universities Meson Facility) is a subatomic physics research laboratory located within the campus of the University of British Columbia in Vancouver. At its heart is a cyclotron which accelerates protons through an air-free chamber between the poles of an electromagnet, the field guiding them in an expanding spiral path. The particles are accelerated by pulses of voltage twice per cycle producing approximately $10^{15} 500 \mathrm{MeV}$ protons per second. When this particle beam reaches the outside of the tank, it is bent into beam lines leading to experimental halls which contain various types of target which, when struck by a proton stream, create an intense beam of various particles for use in related experiments. One of these halls is known as the Thermal Neutron Facility (TNF) which is the final beam dump. This facility is used for testing when a neutron environment is favoured, such as that on board an aircraft where instrumentation and crew can be subject to exposure by neutrons of cosmic origin. It is one of the intentions of the TRIUMF facility that this neutron field should mimic the effect of being at aircraft altitudes in terms of the energy spectrum of the field present ${ }^{(3)}$. It is thought that 1 hour exposed to the output of the TNF is equivalent to around 1,000,000 hours of actual flying at typical altitudes, although this is currently still an approximation made by staff at the facility as opposed to anything verified by scientific methods. A photograph of the TRIUMF facility is shown in Figure 1 although large volumes of shielding obscure much of the facility for safety reasons.

In this report, the results of simulations of the neutron field within the neutron hall at TRIUMF using MCNPX version 2.6.0 are compared to both a theoretical neutron field due to radiation of cosmic origin, and the neutron fluence rate results obtained from the activation of foils which were located at the exit of the facility. The characteristics of several similar facilities and locations have been evaluated in similar ways before, including the laboratory of the nuclear engineering department at the Polytechnic University of Madrid (DIN-UPM) ${ }^{(4)}$, the hadron beam at CERN ${ }^{(1)}$, the high-energy reference field CERF ${ }^{(5)}$, Zacatecas, Mexico $\left(2420 \mathrm{M}\right.$ above sea level) ${ }^{(6)}$, the neutron field at JOYO in Japan ${ }^{(7)}$ and the Sigma Sigma facility in Bucharest ${ }^{(8)}$. However, detailed simulations of the TRIUMF Neutron facility (TNF) have not been published before. 


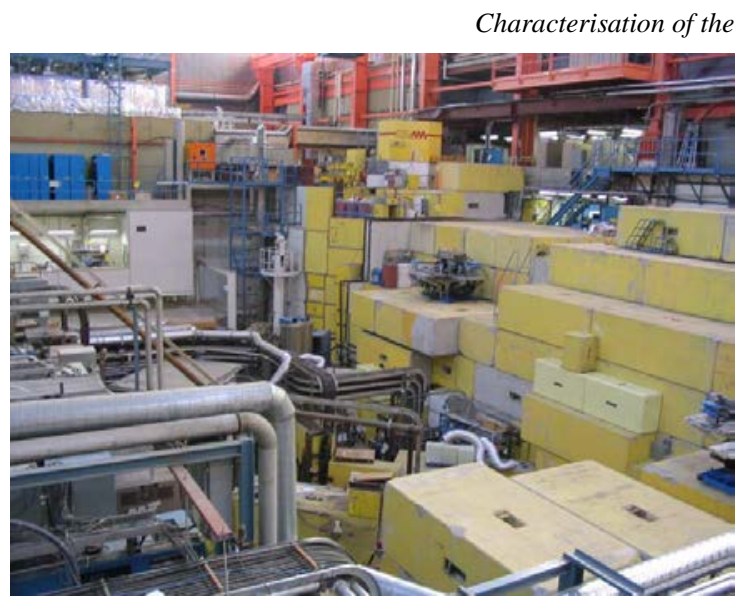

Fig 1: The TRIUMF neutron hall.

\section{THE THERMAL NEUTRON FACILITY GEOMETRY}

The neutron hall section of the TRIUMF facility is the final destination of the cyclotron-generated proton beam. The hall is based around a horizontally-aligned cylindrical aluminium target (with water cooling channels) submerged in a verticallyaligned, cylindrical tank of water. A simulation of the facility was made in 2002 using the FLUKA code (version 1999), although the licence agreement for the code states that:

“All FLUKA versions older than Fluka2008.3d and starting since 1989, are declared obsolete and will no longer be supported. Therefore they shall no longer be used for any publication according to the FLUKA User License."(9)

Since the 1999 version of FLUKA was released there have been various changes and updates such as new cross section libraries which have been completely changed. Further, in FLUKA version 99, the description of thermal neutrons is severely limited. Thus it would be unfair to compare a modern version of an alternative code when the more up to date version of FLUKA is available. However, the geometry and other simulation information from this original FLUKA simulation was utilized in creating the MCNPX model, as the FLUKA version was made by the people who work at the TRIUMF facility and so have the most intimate knowledge of the location. The geometry used within the TNF model was simplified so it consists of just 5 cylinders and 3 orthogonal box shapes. The MCNPX code is written using cm as units of distance and so all co-ordinates referred to here are thus in these units rather than the SI units, millimetres. Also, the following lists of what is contained in the various materials are in terms of molecular proportions rather than mass proportions (i.e water is simulated as having $66.7 \%$ hydrogen as for every oxygen molecule there are two hydrogen molecules).

The Source - The proton source which enters the TNF is modelled as a beam with a geometrical cross section shaped as a circle with a radius of $1.5 \mathrm{~cm}$ and consisting of monoenergetic protons of $450 \mathrm{MeV}$. This latter estimate was made by the staff at the TRUIMF facility when the initial calculations were made when constructing the facility. It is not clear whether this figure was produced using software such as the "Stopping and Range of Ions in Matter" software (SRIM) ${ }^{(10)}$. Although the exact proton fluence rate entering the TNF is not known, the current is estimated to be $140 \mu \mathrm{A}$. If every proton was said to have a charge of $1.602 \times 10^{-19} \mathrm{C}$, this indicates a proton fluence rate of $8.74 \times 10^{14}$ protons per second which is slightly lower than the official estimate of $10^{15}$ protons per second leaving the cyclotron. Using this factor allows the use of units $\mathrm{cm}^{-2} \cdot \mathrm{s}^{-1}$. MeV $\mathrm{Me}^{-1}$ allowing for easy comparisons to cosmic fluence and foil activation data. Within the MCNPX environment this source is modelled as being located within the proton beam line (referred to as cylinder 5 in this document) at $(0,0,74.5)$ and directed mono-directionally along the positive direction on the $z$-axis towards the target (cylinder 1$)$ located at $(0,0,75)$.

Cylinder 1 - The target - This is a horizontally-aligned, cylindrical, aluminium target $50 \mathrm{~cm}$ long with a radius of $10 \mathrm{~cm}$ featuring 13 cooling water channels ${ }^{(11)}$. In order to simplify the model, the target is modelled as being made of a water/aluminium compound (in line with the original FLUKA version) with a density of $2.654{\mathrm{~g} . \mathrm{cm}^{-3}}^{-3}$ and in molecular terms consisting of: 
- $\quad 92 \%$ aluminium-27

- $\quad 2.5 \%$ oxygen- 16

- $\quad 5.5 \%$ hydrogen-1

The centre of one end of this cylinder is located at $(0,0,75)$ with a central vector of $(0,0,50)$ and a radius of $10 \mathrm{~cm}$. This is the first object that the proton field comes across and it is anticipated that nuclear spallation reactions will cause neutrons to be ejected from it.

Cylinder 2 - Water tank - This is a vertically-aligned, cylindrical water tank with a depth of $150.8 \mathrm{~cm}$ and radius of 36.5 $\mathrm{cm}$. The water is modelled as having a density of $1.0 \mathrm{~g} . \mathrm{cm}^{-3}$ and consists (in molecular terms) of:

- $\quad 33.3 \%$ oxygen-16

- $\quad 66.7 \%$ hydrogen-1

The base of the cylinder is centred at $(0,-50.8,100)$ with a central vector of $(0,150.8,0)$ and a radius of $36.5 \mathrm{~cm}$. This water tank therefore contains the target (cylinder 1). It is anticipated that this water will thermalize the majority of the neutrons produced in this target.

Cylinder 3 - Iron shielding - A vertically-aligned cylinder of pure iron (mass number 56) with a density of 7.5 g. $\mathrm{cm}^{-3}$ comprises part of the shielding arrangement. The base of the cylinder is centered at $(0,-149,100)$ with a central vector of $(0,250,0)$ and a radius of $100 \mathrm{~cm}$. This layer of iron surrounds the water tank (cylinder 2).

Cylinder 4 - Concrete shielding - A vertically-aligned cylinder consisting of concrete with a density of 2.35 g. $\mathrm{cm}^{-3}$ a height (length) of $252 \mathrm{~cm}$ and a radius of $260 \mathrm{~cm}$ comprises the other aspect of the shielding set up. The concrete (molecularly) consists of:

- $\quad 55 \%$ oxygen-16

- $24 \%$ silicon-28

- $3 \%$ calcium-40

- $\quad 2 \%$ aluminium-27

- $\quad 16 \%$ hydrogen-1

The centre of the cylinder base is at $(0,-150,100)$ with a central vector of $(0,252,0)$ and a radius of $260 \mathrm{~cm}$. This layer of concrete surrounds the iron layer (cylinder 3), and provides another layer of shielding additional to that of cylinder 3.

Cylinder 5 - Proton beam line - This is modelled as a cylinder containing an absolute pressure of 10,132.5 Pa (corresponding to a density of 0.00012 g. $\mathrm{cm}^{-3}$ ) which contains the proton source and is modelled as a circle as mentioned above. This cylinder has a length of $11 \mathrm{~cm}$ and a radius of $8 \mathrm{~cm}$. The centre of the base of this shape is located at $(0,0$, $64.5)$ and has a central vector of $(0,0,11)$. In practice, this means the proton beam line spans the distance between the edge of the water tank and the end of the aluminum/water target.

Box 1 - Neutron beam line - A rectangular channel of air under vacuum (absolute pressure of 10,132.5 Pa corresponding to a density of 0.00012 g.cm $\mathrm{cm}^{-3}$ as in cylinder 5) with a length of $235 \mathrm{~cm}$, width of $20.8 \mathrm{~cm}$ and a height of $8 \mathrm{~cm}$ constitutes the neutron beam line. This shape represents the channel which transports the neutrons towards the test area. The air under vacuum in the line is modeled in molecular terms as

\footnotetext{
- $\quad 78 \%$ nitrogen-14

- $\quad 21 \%$ oxygen-16

- $1 \%$ argon-40
}

The channel used is located between $26.2 \mathrm{~cm}$ and $34.2 \mathrm{~cm}$ below the bottom of the target and protrudes from the water tank at an angle of $60^{\circ}$ relative to the path of the incident proton beam. In the Monte Carlo models used in this work, the corner of the box is modeled as being at $(35.1,-34.2,109.9)$ with edge vectors of $(195.5,0,113),(-9,0,15.6)$ and $(0,8,0)$. 
Box 2 - Neutron beam line shielding - The neutron channels described in the 'Box 1' section, are all surrounded with a rectangular box of the same iron as in 'Cylinder 3' above. Similarly to box 1, it has a length of $235 \mathrm{~cm}$, but a width and height of $120 \mathrm{~cm}$. The corner of the box is at $(-6,-60,176.5)$ with edge vectors of $(204,0,118),(69.4,0,-120)$ and $(0,120,0)$.

Box 3 - Universe - The entire geometry is modelled as being contained within an air box with dimensions of 10 metres $\times$ 10 metres $\times 10$ metres. This box has a corner at $(-500,-500,-500)$ and edge vectors of $(1000,0,0),(0,1000,0)$ and $(0,0$, $1000)$. The air is simulated as having a density of $0.0012 \mathrm{~g} \cdot \mathrm{cm}^{-3}$ and containing:-

\section{- $\quad 78 \%$ nitrogen-14 \\ - $\quad 21 \%$ oxygen-16 \\ - $1 \%$ argon-40}

The aluminium/water target (cylinder 1) is located within the water tank (cylinder 2), which in turn is surrounded by cylindrical layers of iron (cylinder 3) and then concrete (cylinder 4). The iron cylinder provides $63.5 \mathrm{~cm}$ of shielding around the curved surface of the cylindrical water tank and a metre of shielding below it. The incoming $450 \mathrm{MeV}$ protons cause spallation reactions within the aluminium/water target producing neutrons which are then moderated within this water tank. At a depth of between 26.2 and $34.2 \mathrm{~cm}$ below the target is the neutron beam line which is under vacuum (box 1) and protrudes from the water tank at an angle of $60^{\circ}$ with respect to the incoming proton beam. This channel is surrounded with a thick steel cover (box 2). There are three further channels under vacuum protruding from the water tank (at angles of $60^{\circ}, 240^{\circ}$ and $300^{\circ}$ relative to the incoming proton beam) although these are redundant, with no access available to them. These channels are modelled within the Monte Carlo models in this work but are not anticipated to alter the output significantly. Obviously there is an entire world outside of this simulation, but details of surrounding objects within this facility are not available and are liable to change every day (people, gangways, portable equipment etc...). The world outside of this geometry has been marked as importance equal to zero within the simulations, thus if a particle enters these areas it will immediately be ignored. A Solidworks ${ }^{\mathrm{TM}}$ illustration of the neutron hall is shown in Figure 2 with the proton beam entering from the left-hand side as shown. Also shown is an illustration of the plan view of the neutron part of the facility in Figure 3.

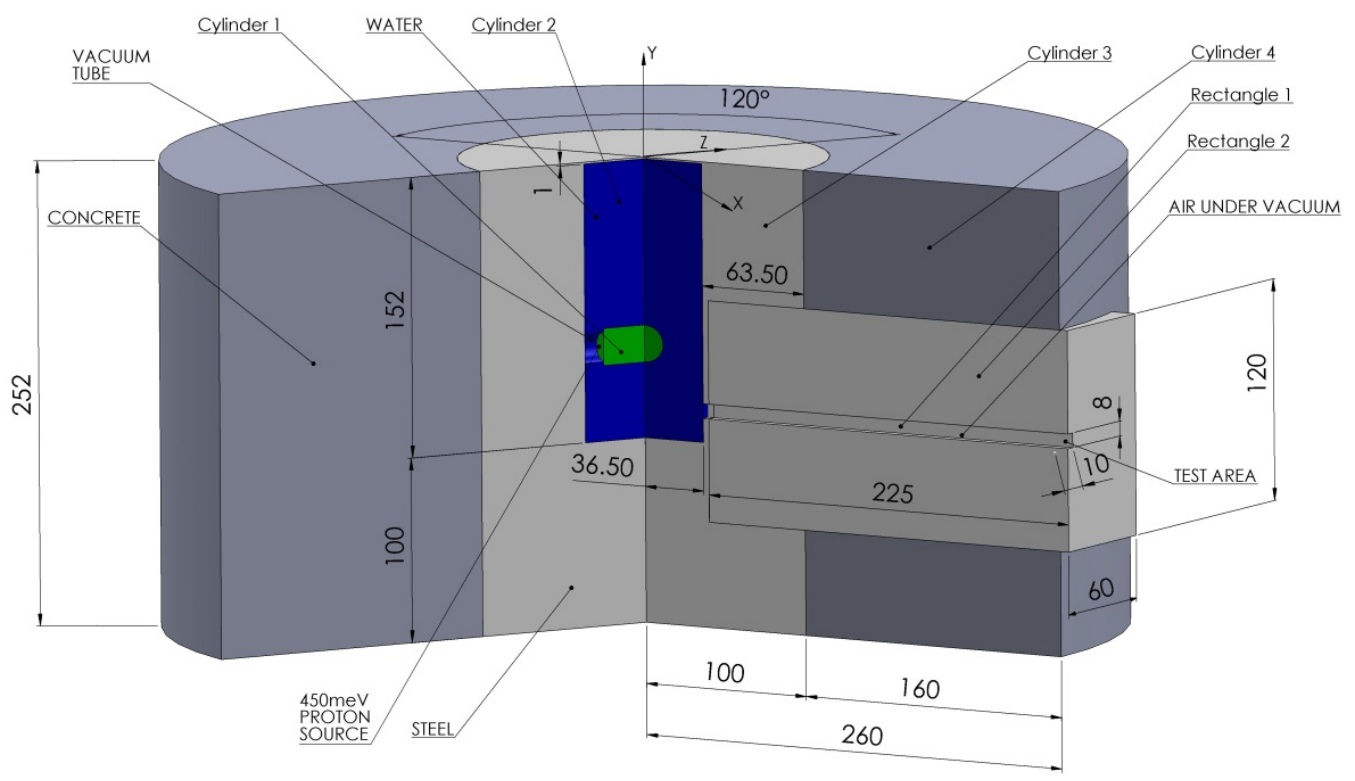


Fig 2: A SolidWorks ${ }^{T M}$ illustration of the TNF.

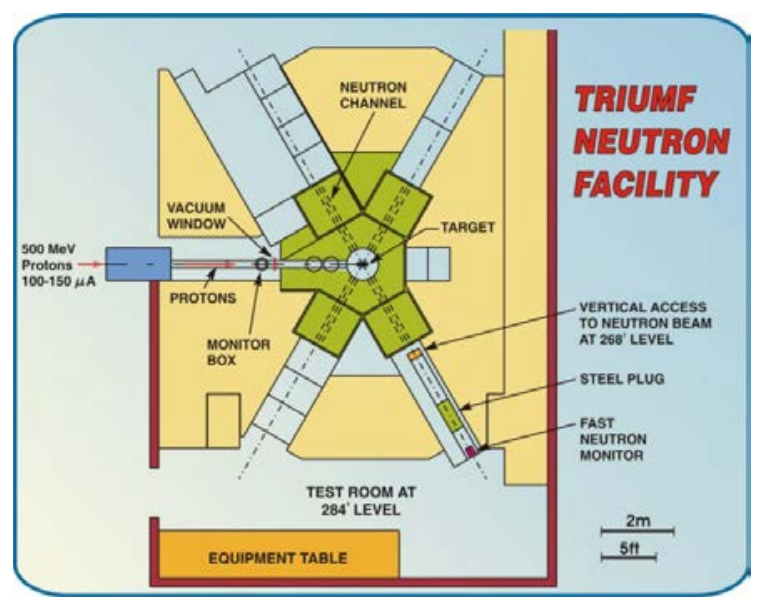

Fig 3: Simplified plan view of the TNF.(23)

\section{SIMULATION SOFTWARE: MCNPX}

The majority of codes developed for particle modelling are based on the Monte Carlo method, renowned for its simplicity of simulation algorithm and the capability to solve complex 3-dimensional problems. MCNPX is a Fortran90-based Monte Carlo transport code which began in 1994 as an extension of MCNP4B and LAHET 2.8, and envisaged as an extension of operability to all particles and energies up to $150 \mathrm{MeV}$. The code relies on numerous 'cards' which are written by the user in order to determine all of the parameters within the MCNPX model such as the physical geometry of the local environment, the source specification and type of result required. MCNPX treats interactions within the environment into categories depending on the energies involved. Tabular data, whose evaluation contains a careful consideration of nuclear structure effects, forms a convenient area of "low" energy phenomena. In the intermediate range, above the nuclear structure region ( $\sim 150 \mathrm{MeV}$ in MCNPX) to a few $\mathrm{GeV}$, the most common modelling methods include intranuclear/ preequilibrium/ evaporation models. At even higher energies, other methods involving quantum effects are used, and so the code contains an early version of the FLUKA code to handle high-energy interactions ${ }^{(12)}$. Current physics modules include the Bertini and ISABEL models taken from the LAHET Code System (LCS), CEM 03, and INCL4. Further, new tally source and variance-reduction options have been developed with libraries for neutrons, photons, electrons, protons and photonuclear interactions. The use of these models can provide the user with control of the physics options. The options controlling the Bertini and ISABEL physics modules are taken from the User Guide to $\operatorname{LCS}^{\mathrm{TM}}{ }^{(13,14)}$. The Bertini ${ }^{(15)}$ model is the default model within the MCNPX environment and for most applications is accurate enough. However, there are slight differences between the models which mostly concern the interactions between the neutrons and other particles within the environment to be simulated. The ISABEL code is an extension by Yariv and Fraenkel ${ }^{(16,17)}$ of the VAGAS code ${ }^{(18)}$, and the CEM 03 model allows neutrons and protons up to $5 \mathrm{GeV}$ and pions up to $2.5 \mathrm{GeV}$ to initiate nuclear reactions. A full discussion of the differences between these models is featured in the MCNPX 2.6.0 user manual, which is available from the MCNPX home page ${ }^{(12)}$.

\section{RESULT PRESENTATION AND UNITS}

In all of the results presented in this work, the tallies chosen have been of the number of particles present per square centimetre of the measured area, per $\mathrm{MeV}$ of energy bin width. The raw results produced by the Monte Carlo software are stated per initial proton generated, and so these figures are multiplied by the estimated proton fluence rate $\left(8.74 \times 10^{14}\right.$ protons/second) to generate a figure with units of $/ \mathrm{cm}^{2} / \mathrm{s} / \mathrm{MeV}$ figure. 250 million particles were used in each of the simulations in order to reduce errors to acceptable levels. Using one of the cores of an Intel core2 processor running at $3 \mathrm{GHz}$ with $3 \mathrm{~Gb}$ of RAM, this corresponded to about 9 days of time running each one. Also, weight window cards were used in order to provide variance reduction in the model and increase accuracy of results further. 
Throughout all of the simulation results here, every effort has been made to present them in as clear a way as possible. In any report which describes a large volume of data, it is important to show the most important aspects of the data clearly. It had been intended that displaying the data in the form of a histogram would be the most suitable thing to do, with a 'stairs' configuration. However, difficulties in plotting the errorbars in an effective manor within the MATLAB r2010b environment ${ }^{(19)}$ ensured that a line plot was settled upon. This output produced this way is very similar to the "stairs" mode of the histogram plot due to the fact that if the $x$-axis is plotted logarithmically, then the points are of equal distance apart and the bin widths are all the same in this mode of axis. A common type of plot is plotting the "fluence per unit lethargy" where the $x$-axis is energy presented in logarithmic units, but the $y$-axis being that shown in equation 1 :

$Y=\frac{F I(E) *\left(E_{\max }-E_{\min }\right)}{\log \left(\frac{E_{\max }}{E_{\min }}\right)} \quad$ (equation 1)

However, in the case of the work presented here, the ratio of Emax to Emin is always 1.3 (keeping consistency with the original FLUKA simulation). Thus the fluence per unit lethargy technique of data presentation is not used as this method does not provide any additional clarity.

\section{RESULTS AND DISCUSSION}

\section{The neutron fluence rate at three locations within the facility}

The geometry described above was defined within the MCNPX 2.6.0 environment, with the neutron fluence rate determined at three locations within the simulated environment defined below and as shown in Figure 4.

- $\quad$ The outside of the target (Cylinder 1)

- $\quad$ The start of the neutron line (Box 1)

- $\quad$ The end of the neutron line (Box 1)

The neutron fluence rate at the surface of the target and the start of the neutron line were simple surface tallies (F2 tally in MCNPX), whereas the tally used to evaluate the fluence rate at the exit of the neutron line was an F4 tally which concerns the average fluence rate across a cell. This was to ape the prior FLUKA simulation which was concerned with the fluence rate across a cell which protruded $10 \mathrm{~cm}$ (away from the facility) from the output of the neutron channel. Figure 5 illustrates the the neutron fluence rate on the surface of the target (cylinder 1) when the rest of the facility is not present (i.e just the proton source and the target are included in the model). This indicates the number of neutrons which are produced by the spallation reaction and ignores neutrons which have been thermalised by the water before returning to the target. Figure 6 shows the fluence rate at the exit of the neutron channel (where the experimental equipment used at the facility is placed) along with a reference cosmic neutron spectrum generated with Qinetiq's QARM program ${ }^{(20)}$. This was calculated at being 10,000 metres above Lancaster, $\mathrm{UK}\left(54^{\circ} \mathrm{N}, 3^{\circ} \mathrm{W}\right)$, and has been amplified by a factor of 1,000,000 in this graph as that has been the factor mentioned previously (i.e. the theory that 1 hour in the output of the TNF facility is equivalent of $1,000,000$ hours at aircraft altitudes).

The thermalised shape of the neutron fluence spectrum at the surface of the target may be unexpected, as at this point the neutrons will not have experienced much water-based moderation and so a less thermalised energy spectrum might be anticipated. Also, the profile of the energy spectrum at the start of the neutron line is virtually the same as that exiting the target, i.e. traversing the water has apparently not thermalised the neutrons further. However, this can be explained by comparing the simulated neutron energy spectrum at the surface of the target with that if the rest of the facility was not there (i.e. just the source and target are modelled) as shown in figure 5. What can be observed here is that the neutron spectrum produced when the proton source has traversed the aluminium target is slightly thermalised, whereas the spectrum produced once the neutrons have returned to the target having scattered back through the water is far more so. Indeed if a "thermalisation ratio" is defined as in equation 2, then in the case of the Proton source just interacting with the target, this figure is 4.19. However if the rest of the facility is modelled too this value jumps to 17,880 - a huge increase illustrating the large number of neutrons being thermalized through the water and scattering back to the target surface.

$$
T R=\frac{1000 \times \text { Fluence rate }(0 \text { to } 1 \mathrm{eV})}{\text { Fluence rate }(1.8-2.35 \mathrm{MeV})}(\text { equation } 2)
$$


The neutron fluence at the entrance to the neutron line is highly thermalized as the neutrons produced within the target have traversed a significant volume of water. Similarly, the neutron fluence at the exit of the neutron line illustrates a similarly thermalised field, although it could be noted the errors have become more significant as the neutrons have interacted with the iron shielding which surrounds the neutron line under vacuum (it would be anticipated that the vacuum within the neutron line would cause virtually no change to the neutron energies due to lack of interactions). The errors are more significant at the lower energy end of the spectrum, probably due to sampling errors brought on by relatively low numbers of neutrons in the energy 'bins' at those locations. This might seem counter intuitive, but there are a small numbers of neutrons at this lower energy range spread over far smaller energy ranges and thus the fluence per MeV of 'bin-width' is comparatively high.

The calculated neutron fluence rate at the end of the neutron line is shown in figure 6 along with a simulated neutron field of cosmic origin magnified by a factor of $1,000,000$. This appears to indicate that the general shape of the energy spectrum is the same for each of these scenarios although the overall per hour neutron fluence rate at the output of the TNF is significantly greater than even a million hours of flight at an altitude of 10,000 metres. However, the units stated are ' $/ \mathrm{cm}^{2} / \mathrm{s} / \mathrm{MeV}$ ' and so the overall received dose depends on how much of the receiving object in question, is subject to this fluence. The neutrons are only emitted from the facility via a letterbox-shaped extrusion with a cross sectional area of 166 $\mathrm{cm}^{2}$, and thus only a small part of the receiving object (such as the human body or apparatus under study) would be irradiated in this way. This is discussed further in the section entitled 'Effective dose' below.

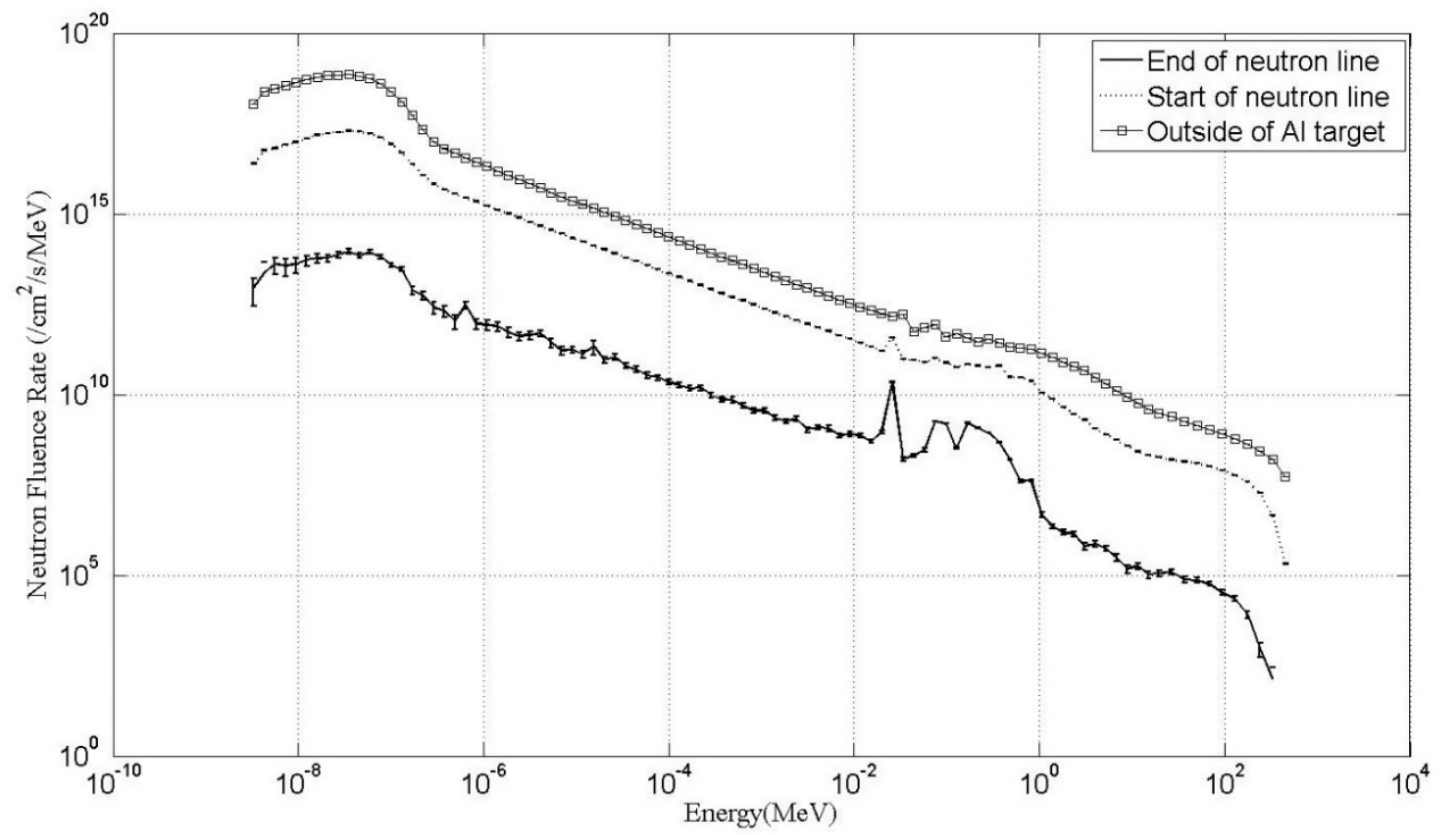

Figure 4: The neutron fluence at three locations within the facility. 
Characterisation of the TRIUMF facility using MCNPX

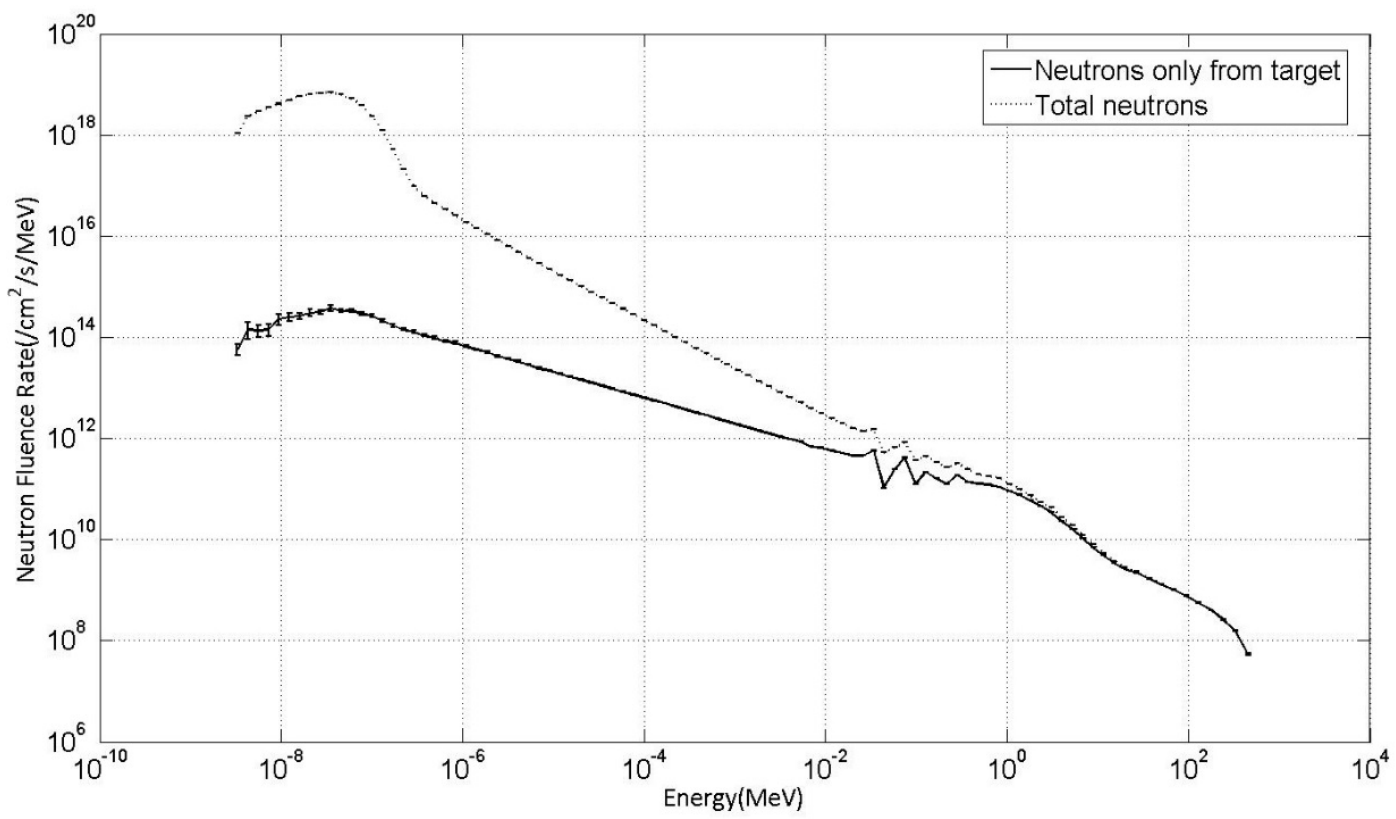

Figure 5: The neutrons on the surface of the aluminium block which have been directly created by the spallation reaction compared to those which have come back after being moderated by the water.

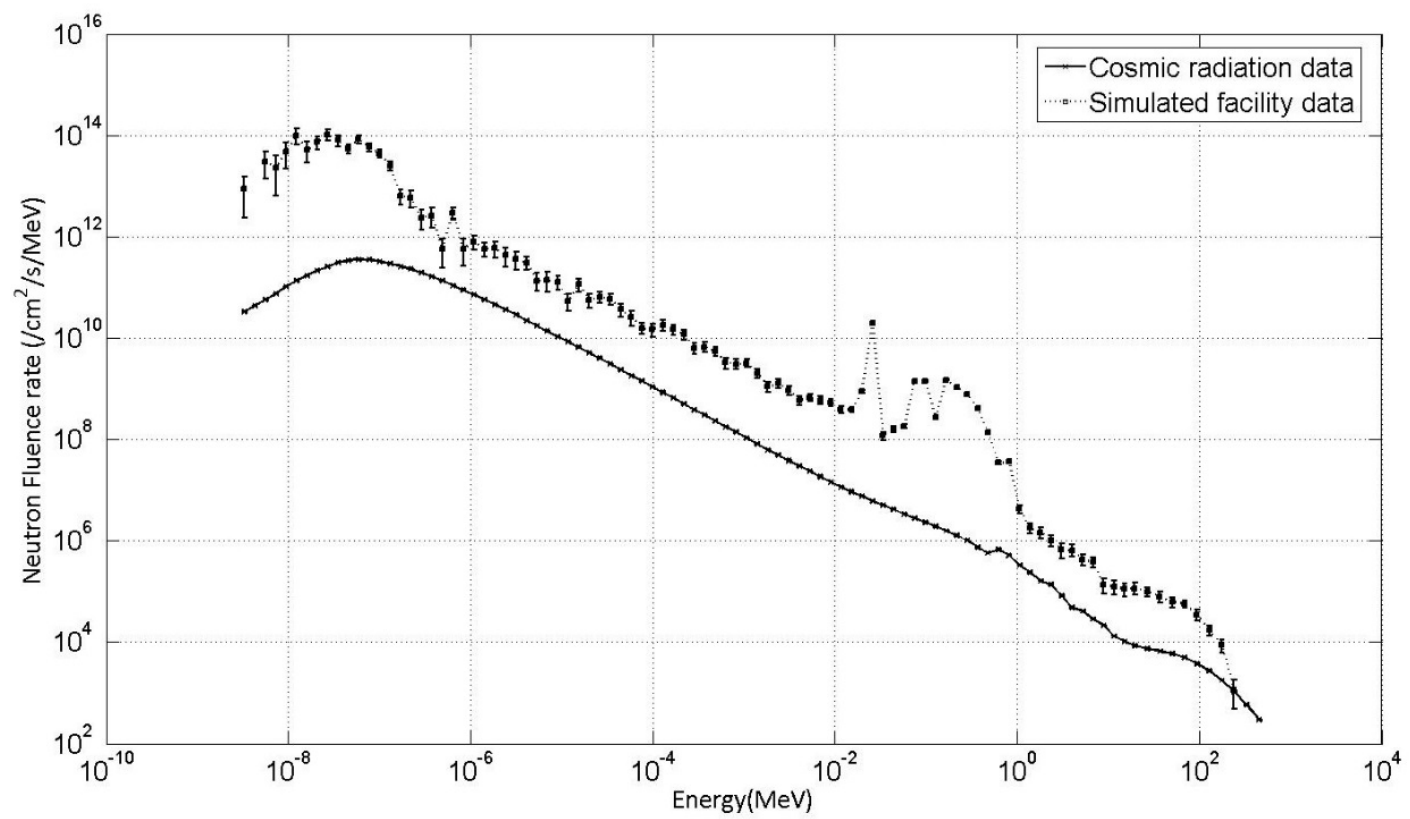

Figure 6: Comparison of the MCNPX calculated output with calculated cosmic radiation data. 


\section{The comparison of four models}

To determine the effects of choosing each of the four main models within the MCNPX environment, the simulations have been run using each of the 4 models available in MCNPX (Bertini, Isabel, Cem 03 and INCL4) in order to determine any differences between them when modelling the facility. These models have been used to simulate the neutron fluence rate at the same three locations as in the "The neutron fluence rate at three locations within the facility" section above. However, for space saving reasons only the fluence rates at the exit of the neutron channel are shown in figure 7 . It was observed that the 4 models were virtually identical (within 1\%) in the other two locations and so there was no advantage in displaying all of the results here. It is clear from these results that the choice of model makes relatively little difference as to the energy spectrum of the neutrons in these simulations. At the surface of the target and the entrance to the neutron line, a significant portion of the neutrons produced will have experienced the conversion from protons via spallation within aluminium, and moderation via water. It would be anticipated that a smaller number of neutrons will have entered the iron shield around the water tank and will have back scattered back into the water - although it is not thought that this would be a significant number. It would be assumed that any deviations between the models when observing these two locations would be due to this scatter effect. The neutron energy spectrum at the exit of the neutron line however, does illustrate a slight deviation between the models as shown in figure 7. However, the differences are still slight, and one can clearly see the relative lack of differences between the 4 models in this graph. Also of interest is the fact that the 3 peaks at an energy of between 10 $\mathrm{keV}$ and $1 \mathrm{MeV}$ still show up consistently across the four models.

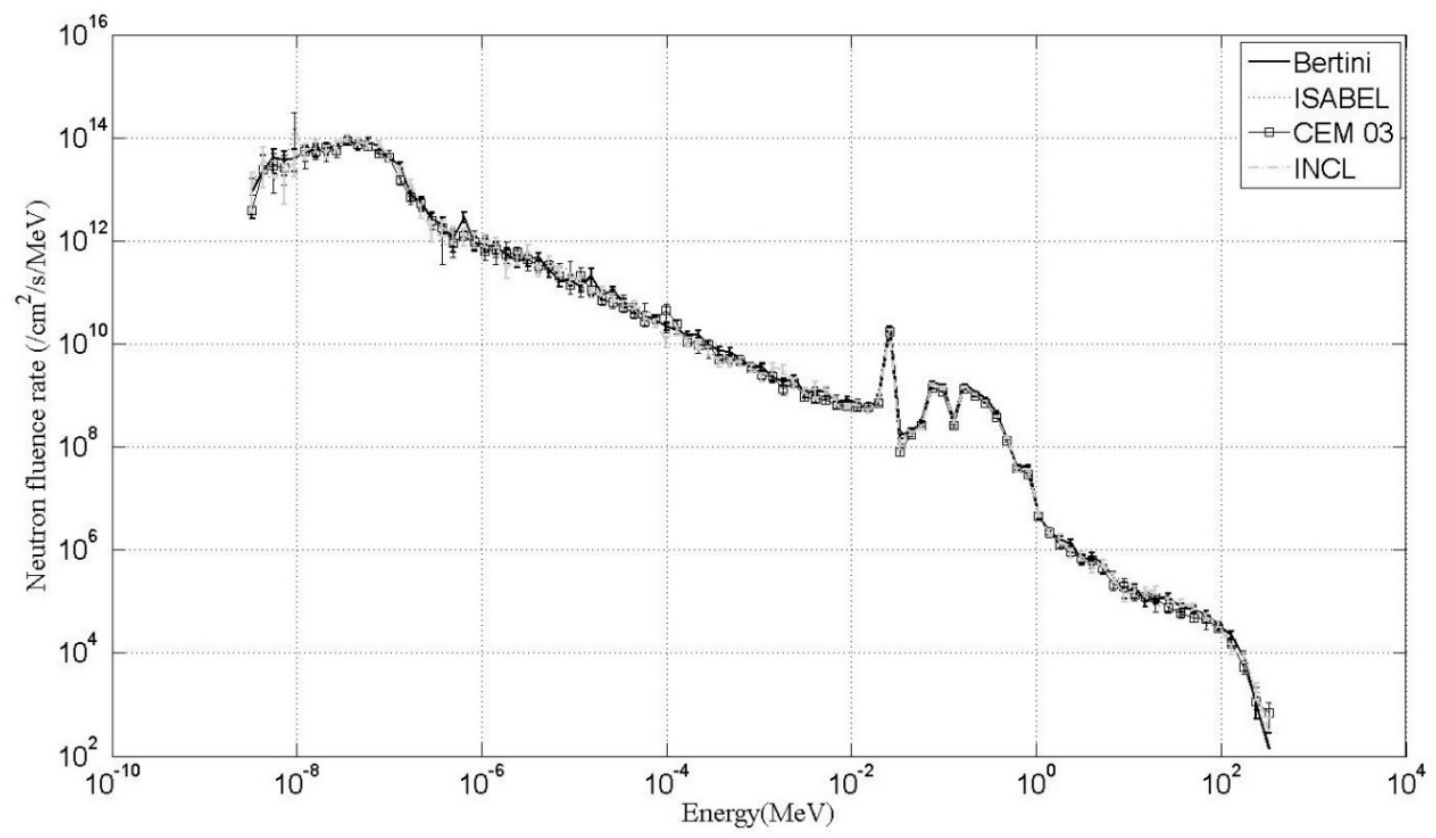

Figure 7: The simulated neutron fluence at the exit of the neutron channel calculated by 4 different models.

\section{The treatment of water in the simulations}

One of the components of thermal scattering is "incoherent inelastic", where the neutrons exchange energy with the target molecule or crystalline lattice and all the scattered waves are assumed to combine incoherently without interference effects. This component is described in terms of a scattering function, $S(\alpha, \beta)$ where $\alpha$ and $\beta$ are reduced values for momentum transfer and energy transfer respectively ${ }^{(21)}$. There is provision within the MCNPX 2.6.0 environment to take account of this behavior in terms of the neutrons being scattered by the hydrogen, via 'Iwtr' cards, the exact one chosen depending on the temperature of the water in question. The 'Iwtr.01t' card assumes a water temperature of $300 \mathrm{~K}$ while 'Iwtr.05t' assumes a temperature of $800 \mathrm{~K}$. As the temperature of the water within the TNF facility is unknown it would seem logical 
to run the simulation using both extremes to further determine the effect of temperature of the water within the simulation and ascertain whether this unknown variable will affect the simulation accuracy. The two sets of results are compared in Figure 8 along with a version where no card is used to take account of this kind of scattering. It can be seen that, generally, the differences between treatments are slight at higher energy ranges (roughly above $10 \mathrm{eV}$ ), but more significant below this - a region roughly corresponding to the thermal region. This is to be expected as the effect is only a low-energy phenomena and the cross sections files provided are for neutrons of $4 \mathrm{eV}$ and less. Very few of the neutrons which leave the exit of the neutron line (2.3\% of the total) are within this $<10 \mathrm{eV}$ range and so it would be concluded, unsurprisingly, that this $S(\alpha, \beta)$ effect is relatively insignificant in these calculations.

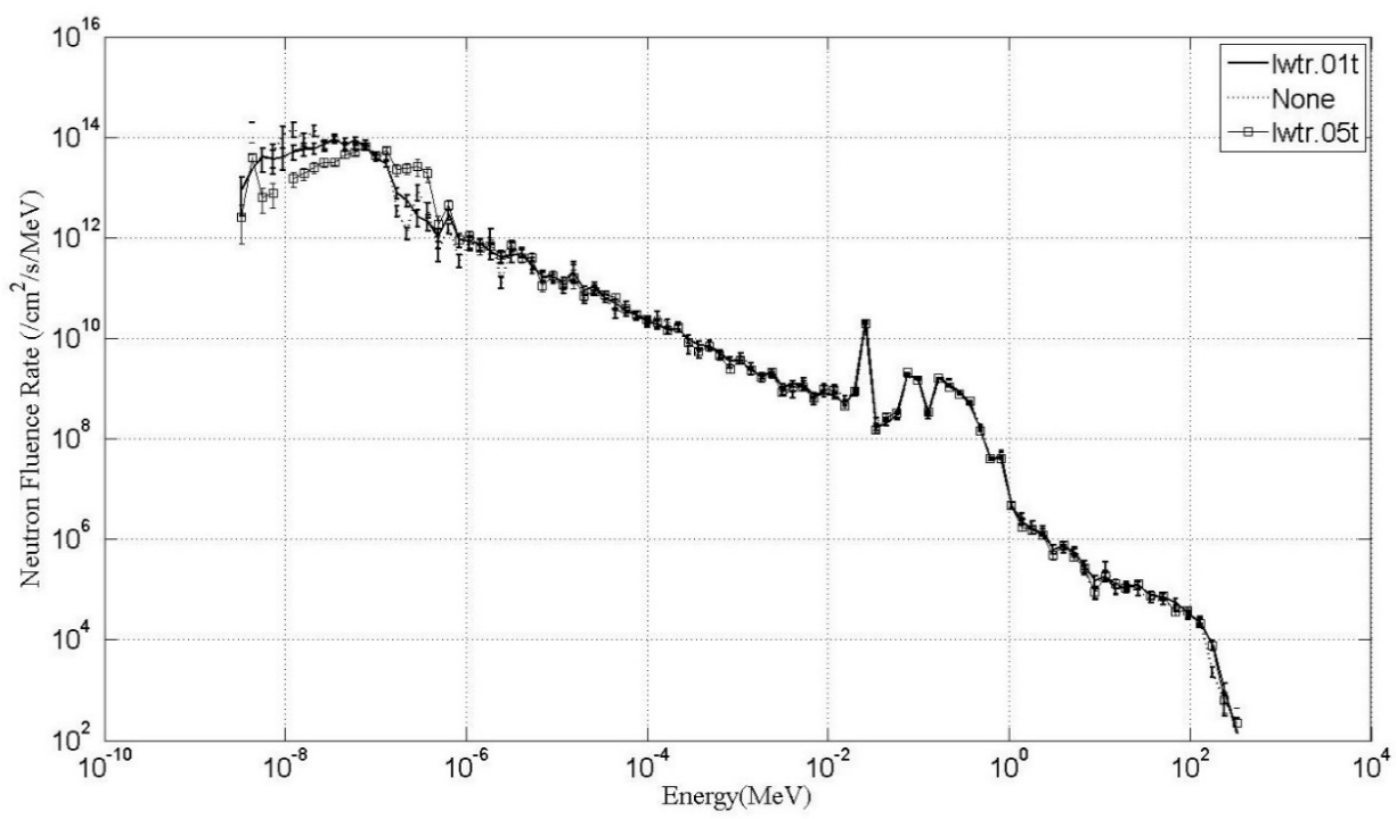

Figure 8: The neutron fluence at the exit of the neutron channel when the lwtr.01t and lwtr.05t functions are used.

\section{Effective dose}

Annex J of ICRP publication $119^{(22)}$ supplies information which can be used to convert neutron fluence to neutron effective dose, for energies up to $180 \mathrm{MeV}$. The neutron spectrum from cosmic origins was calculated via the QARM website ${ }^{(20)}$ (at $10 \mathrm{~km}$ above Lancaster), and using this fluence-todose conversion approach, the effective dose for the neutrons between $0.0033 \mathrm{eV}$ and $175 \mathrm{MeV}$ was calculated to be $2.25 \mu \mathrm{Sv} / \mathrm{hr}$. The neutron component of cosmic radiation tends to make up roughly $50 \%$ of the total effective dose, and thus this figure would appear to be feasible. The rough approximation of 1 hour in the TNF facility being equivalent to 1 million hours on a commercial aircraft ensures that 1 million hours would equate to a dose of $2.25 \mathrm{~Sv} / \mathrm{hr}$. Treating the MCNPX-derived TNF facility results the same way produces an equivalent figure of $135 \mathrm{~Sv} / \mathrm{hr}$; a far higher figure. However, this assumes that the whole body would be subject to the same level of radiation, in the way it is assumed that cosmic radiation is constant across the body. However, the neutrons leave the facility via a "letterbox" shape with dimensions of $20.8 \mathrm{~cm}$ wide by $8 \mathrm{~cm}$ high; i.e. a total cross section of $166 \mathrm{~cm}^{2}$. Taking the body to have a surface area of $1.85 \mathrm{~m}^{2}$ (assuming a height of $1.71 \mathrm{~m}$, a weight of $73 \mathrm{~kg}$ and using the Dubois and Dubois formula ${ }^{(23)}$ ), this would indicate that the effective neutron dose due to the facility would only be $1.21 \mathrm{~Sv} / \mathrm{hr}$ if only the neutrons output from the letterbox section were considered. This is within an order of magnitude of the figure of 2.25 Sv/hr simulated as being the neutron effective dose of 1,000,000 hours at an altitude of 10,000 metres.

\section{Practical work using foils}

A number of foil activation measurements have been carried out within the TNF facility using nickel, aluminium and carbon foils to measure the $>1 \mathrm{MeV}$ neutron component, with gold and indium foils used to measure the thermal neutron 
component ${ }^{(3)}$. The results obtained for the nickel, aluminium and carbon foils with the equivalent MCNPX values are (total neutron flux between 1 and $400 \mathrm{MeV}$ ):

- $\quad$ Nickel $-5.6 \times 10^{6} \mathrm{~cm}^{-2} \mathrm{~s}^{-1}$

- Aluminium $-7.2 \times 10^{6} \mathrm{~cm}^{-2} \mathrm{~s}^{-1}$

- Carbon $-5.6 \times 10^{6} \mathrm{~cm}^{-2} \mathrm{~s}^{-1}$

- $\operatorname{MCNPX}(1$ to $400 \mathrm{MeV})-11.9 \times 10^{6} \mathrm{~cm}^{-2} \mathrm{~s}^{-1}$

- $\quad$ TNF website thermal neutron fluence rate estimate $-5.0 \times 10^{5} \mathrm{~cm}^{-2} \mathrm{~s}^{-1}$

- $\quad$ MCNPX (thermal neutrons) $=6.38 \times 10^{5} \mathrm{~cm}^{-2} \mathrm{~s}^{-1}$

The results for the gold and indium foils are not published here in the same format as they are for these materials. However, the TNF part of the TRIUMF website ${ }^{(24)}$ reports that their own estimation of thermal neutron fluence rate is $5 \times 10^{5} \mathrm{~cm}^{2} \cdot \mathrm{s}^{-1}$. It is not immediately clear what the researchers consider to be thermal (i.e how far from the standard value of $0.025 \mathrm{eV}$ is considered thermal). However the MCNPX simulation has determined that the exit of the neutron channel would feature $6.38 \times 10^{5} \mathrm{~cm}^{-2} \mathrm{~s}^{-1}$ between the energy range of $0.0207 \mathrm{eV}$ and $0.0269 \mathrm{eV}$. The authors make many assumptions in the work and it is not possible to determine whether the ratios that arise would still hold true in the final figure if they calculated it in the same way as for the three foils intended to indicate the higher energy band. The results presented in this work indicate that the simulated neutron count is consistent with the count predicted with the foils, considering standard error propagation throughout the calculations and simulations. The origin of such errors in these calculations and this work in general includes:-

- The MCNPX specifications such as the energy of the incoming protons, and the current are both estimations.

- $\quad$ The magnitude of the vacuum in the proton and neutrons channels is estimated.

- The errors within the MCNPX simulations.

- The rest of the facility outside of the stated geometry not being modelled (people, gangways etc...).

- The uncertainty over exact neutron energies within the bins when converting to effective dose.

- The assumptions in the foil analysis taken to produce suitable output figures.

- The errors usually associated with the use of activated foils as neutron field analysis.

\section{CONCLUSION}

The general trends indicated by all of the simulations featured in this work reflect that the neutron field at the exit from the TNF within the TRIUMF facility mimics those of a typical cosmic neutron spectrum. The neutron hall is designed to mimic approximately a million hours within a cosmic radiation field. This is consistent (within an order of magnitude) if only the 'letterbox' area which emits the neutrons is taken into account, which appears to be a fair assumption. Up to an energy of $175 \mathrm{MeV}$, the effective dose due to 1 million hours of cosmic radiation is calculated to be $2.25 \mathrm{~Sv}$ compared to a value of $1.21 \mathrm{~Sv} / \mathrm{hr}$ at the exit of the TNF. There are other slight differences between the two spectra such as the lack of increased numbers of particles with energies of around $1 \mathrm{MeV}$ and $100 \mathrm{MeV}$ as would usually be found within a neutron beam of cosmic origin. Neutron data obtained using various activation foils was also compared within this work in order to provide a practical set of results for comparison purposes. Again, within an order of magnitude, these values agree with the simulated results obtained with the Monte Carlo software.

\section{ACKNOWLEDGEMENTS}

We acknowledge Ewart Blackmore and Lutz Moritz for their help in characterising the field and providing information relating to the FLUKA reconstruction and for permission to reproduce original illustrations. Also thanks to the Nuclear Decommissioning Authority for their support.

\section{REFERENCES}

1. Birattari, C. De Ponti, E. Esposito, A. Ferrari, A. Pelliccioni and M. Silari, M. Measurements and characterization of high energy neutron fields, Nucl. Instrum. Meth. Phys. Res. A, 338 534-543 (1994)

2. Birattari, C. Esposito, A. Fasso, A. Ferrari, A. Festag, J.G. Hofert, M. Nielsen, M. Pelliccioni, M. Raffnsoe, C. Schmidt, P. and Silari, M. Intercomparison of the response of dosimeters used in high-energy stray radiation-fields Rad. Prot. Dos. 51 (2) 87-94 (1994)

3. Blackmore, E.W. and Kopetski C. Use of the TNF neutron beam for radiation damage studies, Design note, TRI-DN-02-16 (2002) 
4. Gallego, E. Lorente, A. and Vega-Carrillo, H.R. Characteristics of the neutron field of the facility at DIN-UPM, Rad. Prot. Dos. 110 (14) $73-79$ (2004)

5. Mayer, S. and Otto, T. Investigations of a recombination chamber as a mixed field dosimeter in comparison with a tissue-equivalent proportional counter at CERF, Nucl. Instrum. Meth. Phys. Res. A, 505 399-402 (2003)

6. Vega-Carrillo, H.R. and Manzares-Acuna, E. Background neutron spectrum at 2420m above sea level Nucl. Instrum. Meth. Phys. A, 524 146-151 (2004)

7. Aoyama, T. Sekine, T. and Tabuchi, S. Characterisation of neutron field in the experimental fast reactor JOYO for fuel and structural material irradiation test Nucl. Eng. Des., 228 21-34 (2004)

8. Angelescu, T. Ghiordanescu, A. Mihul, A. Garlea, A. Garlea,I. Labau,V. Vasilescu,A. Beregic,V. and Baltateanu,N. Gamma dose characterisations of the neutron field in the Sigma Sigma irradiation facility Nucl. Instrum. Meth. Phys. Res. A, 378 594-597 (1996)

9. Statement of FLUKA obsoleteness ( $3^{\text {rd }}$ March, 2014) found at http://www.fluka.org/fluka.php?id=release_notes\&mm2=3

10. SRIM Website ( $3^{\text {rd }}$ March 2014) found at http://appdb.winehq.org/objectManager.php?sClass=application\&iId=5992

11. Hodges, T.A. Langstaff, R.R. and Lenckowski, M. An aluminium plate beam dump for the TNF in BL1A, VPN, internal document 2 (1995)

12. MCNPX website ( $3^{\text {rd }}$ March 2014) found at http://mcnpx.lanl.gov/

13. Prael R.E.and Lichtenstein,H. User Guide to LCS: The LAHET Code System, Los Alamos National Laboratory report LA-UR-89-3014, Revised (September 15, 1989).

14. User guide to LCS ( $3^{\text {rd }}$ March 2014) found at http://library.lanl.gov/cgi-bin/getfile?00208689.pdf

15. Bertini,H.W. Intranuclear-Cascade calculation of the secondary nucleon spectra from nucleon-nucleus interactions in the energy range 340 to $2900 \mathrm{MeV}$ and comparisons with experiment, Phys. Rev. 188(4) 1711-1730 (1969)

16. Yariv,Y. and Fraenkel,Z. Intranuclear cascade calculation of high-energy heavy-ion collisions - effect of interactions between cascade particles, Phys. Rev. C, 24 (2), 488-494 (1981)

17. Yariv,Y. and Fraenkel,Z. Intranuclear cascade calculation of high-energy heavy-ion interactions, Physical Review C, 20 (6), $2227-$ 2243 (1979)

18. Chen,K. Fraenkel,Z. Friedlan,G. Grover,J.R. Miller,J.M. and Shimalot,Y. VEGAS - A Monte Carlo simulation of intranuclear cascades Phys. Rev. 166 (4), 949 (1968)

19. MATLAB data manipulation software ( $3^{\text {rd }}$ March, 2014) found at: http://www.mathworks.co.uk/products/new_products/release2010b.html

20. QARM website for calculating atmospheric radiation ( $3^{\text {rd }}$ March 2014) found at http://86.27.225.122:8080/qarm/index.jsp?URL=changes.jsp

21. Detail of S(alpha, beta) scattering ( ${ }^{\text {rd }}$ March, 2014) found at http://t2.lanl.gov/nis/njoy/ther02.html

22. ICRP, 2012. Compendium of Dose Coefficients based on ICRP Publication 60. ICRP Publication 119. Ann. ICRP 41(Suppl.).

23. DuBois,D. DuBois,D.F. A formula to estimate the approximate surface area if height and weight be known Arch. Int. Med. 1916;17: pp863-71. (1916)

24. TNF part of the TRIUMF Website (24 $4^{\text {th }}$ April 2014) found at http://www.triumf.ca/pif-nif/nif-beam-specifications-0 